\title{
Assessment of hospital-based adult triage at emergency receiving areas in hospitals in Northern Uganda.
}

\author{
Keneth Opiro ${ }^{1}$, Lee Wallis ${ }^{2}$, Martin Ogwang ${ }^{3}$
}

1. Gulu University Faculty of Medicine, P.O.Box, 166 Gulu Uganda

2. University of Cape Town Faculty of Health Sciences, Surgery, Division of Emergency Medicine

3. St. Mary's hospital Lacor, P. O. Box, 180, Gulu Uganda, Surgery

\begin{abstract}
Background: Limited health service resources must be used in a manner which does "the most for the most". This is partly achieved through the use of a triage system. Whereas efforts have been made to introduce paediatric triage in Uganda such as Emergency Triage Assessment and Treatment Plus (ETAT+), it is not clear if hospitals have local protocols for adult triage being used in each setting.

Objectives: To determine the presence of existing hospital triage systems, the cadre of staff undertaking triage and barriers to development/improvement of formal triage systems.

Methodology: This was a descriptive cross-sectional study. Acholi sub-region was randomly selected for the study among the three sub-regions in Northern Uganda. The study was conducted in 6 of the 7 hospitals in the region. It was a written self-administered questionnaire.

Results: Thirty-three participants from 6 hospitals consented and participated in the study. Only one hospital (16.7\%) of the 6 hospitals surveyed had a formal hospital-based adult triage protocol in place. Only $2(33.3 \%)$ hospitals had an allocated emergency department, the rest receive emergency patients/perform triage from OPD and wards. Lack of training, variation of triage protocols from hospital to another, shortage of staff on duty, absence of national guidelines on triage and poor administrative support were the major barriers to improvement/development of formal triage in all these hospitals.

Conclusion: Formal adult hospital-based triage is widely lacking in Northern Uganda and staff do perform subjective "eyeball" judgments to make triage decisions.

Keywords: Triage, "eyeball" triage, emergency receiving areas, and emergency health conditions.

DOI: https://dx.doi.org/10.4314/ahs.v17i2.23

Cite as: Opiro K, Wallis L, Ogwang M. Assessment of hospital-based adult triage at emergency receiving areas in hospitals in northern Uganda. Afri Health Sci. 2017;17(2): 481-490. https:/ / dx.doi.org/10.4314/abs.v17i2.23
\end{abstract}

\section{Introduction}

Proper decisions have to be made on how to distribute healthcare resources when the demands significantly exceed the available resources. These decisions are made at various levels of health services ranging from government (under the Ministry of Health in its resource alloca-
Corresponding author:
Keneth Opiro,
Emergency Medicine (University of Cape Town),
Division of Emergency Medicine,
University of Cape Town
Private Bag X3
7701 Rondebosch, South Africa
Tel: +256774210558,
Email: opiroken@yahoo.co.uk

tions), to facility based emergency receiving areas where healthcare providers have to decide on who receives immediate emergency care and who can wait when the demand is high. While these decisions are being made at facility based emergency receiving areas, it is important to recognize that not all needs can be satisfied urgently, therefore rationalization using a proper guiding tool in order to maximize benefits of emergency health interventions is very important. It is this guiding tool that is referred to as triage scale. In other words, limited health service resources must be used in a manner which does "the most for the most". This is partly achieved through the use of a triage system, but health workers must understand it and it must be used routinely ${ }^{1}$.

In resource limited settings - especially rural areas such as in Uganda, just like other sub-Saharan African nations, the use of international triage systems may not be appro- 
priate; simple scales may be sufficient to perform its function such as the one developed in South Africa which has been found to be effective in rural areas and now widely implemented even in other countries ${ }^{2,3}$. Having such a scale that is put in use would be a significant milestone in improving emergency care to acutely ill in Uganda.

Emergency care is thought by many resource limited countries as not being cost effective, however, this is demonstrably inaccurate ${ }^{4}$ as simple and cheap tools such as triage scale makes much input in emergency care of patients. A recent survey regarding emergency and surgical care access in sub-Saharan Africa showed that only $19-50 \%$ of hospitals had the ability to provide 24 -hour emergency care and the main reason was infrastructure challenge ${ }^{5}$. However, even with the available infrastructure, improving emergency services is still possible if the quality and timeliness of interventions when very sick patients arrive at emergency receiving areas is improved; one way is the use of simple tools such as triage ${ }^{6}$.

Uganda, just like many sub-Saharan African countries, is experiencing increasing population making it to face challenges with increasing number of both medical emergencies from non-communicable disease ${ }^{7}$ and surgical/trauma related injuries especially from road traffic accidents and its resultant mass casualty incidents ${ }^{8}$. Whereas efforts have been made to introduce paediatric triage in Uganda such as Emergency Triage Assessment and Treatment Plus (ETAT+), the WHO tool and Integrated Management of Childhood Illness (IMCI) ${ }^{9}$, such programs are lacking for adult emergency medical care and it is not clear if hospitals have local protocols for adult triage.

One triage score, the Kampala Trauma Score (KTS), was initially found to be a trauma triage tool for resource poor settings ${ }^{10}$. However, its usefulness was found to be limit$\mathrm{ed}^{11}$. A report from the Malaria Consortium Uganda on a survey done to determine severe malaria clinical practice in Hoima, central Uganda, found that although not specific to adult triage, only $44 \%$ of those health facilities surveyed had established and functional triage systems in place $^{12}$.

In sub-Saharan Africa, the burden of emergency conditions is high, with high mortality from these conditions, there are poor emergency care services mostly due to resource limitations and there is limited research done. In
Uganda specifically, there is very few published research on emergency care and even those available is all rotating around Kampala the capital city, with little if any, known about other regions located in the country side such as Northern Uganda. Hence this study is aimed at assessing the practice of adult hospital-based emergency triage in hospitals in Northern Uganda.[T1]

\section{Methodology \\ This was a descriptive cross-sectional study. Study setting and population}

Northern region is divided into three sub-regions: Acholi, Lango and West-Nile. Each sub-region has a similar distribution of hospitals, with each having one Regional Referral hospital, at least two faith based and at least two district hospitals. The seven hospitals in the sub-region serving population of $2.7^{13}$ million people are: Gulu Regional Referral Hospital (government facility) with emergency unit annual attendance of over 10,000; St. Mary's Hospital Lacor (PNFP) with average annual emergency receiving areas' attendance of about 14,000; Kalongo Hospital (PNFP) with average annual admissions of about 11,500; St. Joseph's Hospital Kitgum (PNFP) with average annual admissions of about 10,000; Anaka hospital and Kitgum District hospital (government facilities), each with same total bed capacities of 250; and Gulu independent Hospital (private for profit) with average annual emergency attendance of slightly above 3,000. (Statistics on hospitals got from personal communication with hospital managers). Participants were health professionals who were involved in daily initial management of emergency patients at the emergency receiving areas of these hospitals. The study was carried out in 6 of the 7 hospitals in the sub-region.

\section{Study site selection}

Allocating numbers to the three sub-regions (Acholi, Lango and West Nile), and using a random number generator, Acholi sub-region was randomly selected for the study.

\section{Sample size}

To seek representativeness and experience from each hospital, at least one representative of nurses in various duty shifts (night, morning and evening shifts), the nursing in-charge/leader, one doctor (head of department or 
any doctor on duty), if available, and a clinical officer if any, making a minimum of 5-6 staff who consented to participate in the study from each hospital were recruited.

\section{Inclusion and exclusion criteria: \\ Inclusion}

-Staff who consented to participate in the survey,

-Health professionals - nurses, clinical officers, and doctors.

\section{Exclusion criteria}

- Those who refused to consent to participate in the study, - Those who were not qualified health personnel (e.g. receptionists, cashiers, cleaners, social workers, porters, etc) as they were not involved in making triage decisions.

\section{Data collection, management and analysis}

This was in the form of self-administered written questionnaire - each participant read and noted his/her response according to instructions. Language used in the questionnaires was English as this is the official language for education in Uganda and all qualified staff are able to read and write. Data collection was done from December 2015 to January 2016. The collected data was compiled and kept under safe custody of the investigator. The information was entered in a work computer and the electronic records were password-protected. Only investigators had access to data. Information entered into Microsoft Excel for Windows, and analysed using Statistical Package for the Social Sciences (SPSS). Descriptive statistics are reported here. Where space was allocated for staff to make comments, these were noted, aggregated, categorized and reported.

\section{Quality control}

Staff were asked to fill questionnaires just before the start of the duty, at break time, or after duty when they had signed out of duty to avoid interference of duty and hurried responses which may affect the quality of the information. Some staff made appointments outside duty hours for the survey. All participants had plenty of time and privacy as required to fill the questionnaires without interference.

The design of the questions was done in such a way as to eliminate desirability bias as well as obtain background information as accurately as possible.
The questionnaires were adopted partly and modified from the study done in Sweden ${ }^{14}$. To test the questionnaire, a pilot study was conducted in Kuluva hospital located in West Nile sub-region Northern Uganda. Two nurses from OPD consented and participated to pretest the questionnaire in terms of readability and ease of understanding. In addition, face to face interactions with them were made to specifically ask for difficulties, if any, in reading and understanding the questionnaires. No difficulties were reported by them and therefore no any change was made on the questionnaires after the pilot study. The questionnaire was adopted and modified by the investigators[T2]

\section{Ethical considerations}

Ethical approval was obtained from the University of Cape Town (HREC No. 705/2015). In Uganda, ethical approval was obtained from local Research and Ethic committee - St. Mary's Hospital Lacor - accredited by Uganda National Council of Science and Technology (UNCST) for ethical approval ${ }^{15,16}$. In addition, individual institutional permission where this study was conducted was sought before collecting data.

There were no health risks, discomforts, or inconveniences reported due to participation. Information obtained from this study will be used to guide further development of adult triage systems which will improve emergency medical care in Northern Uganda and Uganda at large.

Data was kept confidentially. No participants' identifying data were collected. Each participant had the comfort and confidence required during filling of the questionnaires.

Each study participant signed informed consent. They were free to decline from participating in the study and to withdraw at any time with no consequences.

There were no monetary or material forms of reimbursement to participants for taking part in this study.

\section{Results}

Six out of seven hospitals $(85.7 \%)$ granted permission to conduct the research. Overall there were total of 33 (out of 30-36 expected) participants enrolled in the study from all the six hospitals. This included 5 doctors, 4 physician assistants (clinical officers), 11 registered nurses, 9 enrolled nurses and 4 nursing assistants who participated in the study. 
All in all, the majority had at least one-year experience in emergency receiving areas. Most (45.5\%) participants had experience for greater than two years, followed by $1-2$ years $(21.2 \%), 6-12$ months $(15.2 \%)$ and $18.2 \%$ had less than 6 months experience working at emergency receiving areas.

\section{Presence of formal triage protocol}

Only one of the six hospitals $(16.7 \%)$ - St. Mary's hospital Lacor was found to have a formal local triage protocol in use. The other 5 hospitals had no formal triage guidelines and staff applied subjective "eye-ball" triage to judge on which patients needed emergency attention.
Only two hospitals (33.3\%) -St. Mary's hospital Lacor and Gulu Regional Referral Hospital had an allocated emergency department, while in the other hospitals emergency triage was performed from OPD and various hospital wards.

\section{Categories [T4] of cadres performing triage}

In two hospitals -St. Mary's hospital Lacor and Gulu Regional Referral Hospital - all staff (100\%) reported triage was done by enrolled and registered nurses, and all staff in the other four hospitals reported nursing assistants were involved in triage activities in addition to enrolled and registered nurses.

Table 1. Participants' opinion on possible barriers to improving triage in hospital with formal local protocol

\begin{tabular}{|llllll|}
\hline $\begin{array}{l}\text { Limiting factors to improving triage } \\
(\mathbf{n}=\mathbf{5}\end{array}$ & $\begin{array}{l}\text { Strongly } \\
\text { Agree }\end{array}$ & Agree & $\begin{array}{l}\text { Not } \\
\text { Sure }\end{array}$ & Disagree & $\begin{array}{l}\text { Strongly } \\
\text { Disagree }\end{array}$ \\
\hline $\begin{array}{l}\text { Triage scales varies from one hospital to } \\
\text { another }\end{array}$ & $60 \%$ & $20 \%$ & $0 \%$ & $0 \%$ & $20 \%$ \\
$\begin{array}{l}\text { No triage chart displayed in emergency } \\
\text { receiving areas to guide practice }\end{array}$ & $0 \%$ & $0 \%$ & $0 \%$ & $40 \%$ & $60 \%$ \\
$\begin{array}{l}\text { Lack of regular training of staff on triage } \\
\text { Shortage of staff on duty to perform triage }\end{array}$ & $80 \%$ & $20 \%$ & $0 \%$ & $0 \%$ & $0 \%$ \\
$\begin{array}{l}\text { Staff have poor attitudes toward sticking to } \\
\text { triage guideline }\end{array}$ & $60 \%$ & $0 \%$ & $0 \%$ & $20 \%$ & $20 \%$ \\
$\begin{array}{l}\text { No space to allocate patients into } \\
\text { categories even if triaged }\end{array}$ & $40 \%$ & $20 \%$ & $0 \%$ & $0 \%$ & $40 \%$ \\
Poor administrative policy and support & $20 \%$ & $40 \%$ & $0 \%$ & $20 \%$ & $20 \%$ \\
\hline
\end{tabular}

Most participants concurred with the suggested barriers to improving triage except on the points of poor administrative support, poor attitudes of staff, no space allocated for triage and absence of triage chart displayed inemergency receiving areas where some staff disagreed.

\section{Settings with formal triage protocol present}

In St. Mary's hospital Lacor (with formal local triage protocol), three Colour codes were used (red, yellow and green). Two of the five respondents reported that written triage guideline/algorithm was kept on the wall while the rest said they were kept in drawers. All staff reported no formal training on this protocol. All staff rated their local protocol as "good" and acknowledged the importance of improving the current protocol.

\section{Opinion on possible barriers to improving existing} local triage protocol and way forward:

Barriers - all staff agreed with the lack of training as the major barrier, followed by variation of triage scales from one hospital to another and shortage of staff on duty to perform triage. (table 2). 
Table 2. Participants' opinion on the way forward to improve triage in hospitals with formal local protocol

\begin{tabular}{|c|c|c|c|c|c|}
\hline $\begin{array}{l}\text { Way forward to improve triage } \\
(n=5)\end{array}$ & $\begin{array}{l}\text { Strongly } \\
\text { Agree }\end{array}$ & Agree & $\begin{array}{l}\text { Not } \\
\text { Sure }\end{array}$ & Disagree & $\begin{array}{l}\text { Strongly } \\
\text { Disagree }\end{array}$ \\
\hline Conduct regular training on triage & $100 \%$ & $0 \%$ & $0 \%$ & $0 \%$ & $0 \%$ \\
\hline Adopt a unified triage scale nationwide & $100 \%$ & $0 \%$ & $0 \%$ & $0 \%$ & $0 \%$ \\
\hline $\begin{array}{l}\text { Need to assign staff specifically to do } \\
\text { triage during each shift }\end{array}$ & $80 \%$ & $20 \%$ & $0 \%$ & $0 \%$ & $0 \%$ \\
\hline $\begin{array}{l}\text { Print triage tool(chart/scales) and put in } \\
\text { open place in emergency receiving areas }\end{array}$ & $100 \%$ & $0 \%$ & $0 \%$ & $0 \%$ & $0 \%$ \\
\hline $\begin{array}{l}\text { Administrative support in terms of number } \\
\text { of staff on duty }\end{array}$ & $100 \%$ & $0 \%$ & $0 \%$ & $0 \%$ & $0 \%$ \\
\hline Emphasis for staff to stick to guideline & $100 \%$ & $0 \%$ & $0 \%$ & $0 \%$ & $0 \%$ \\
\hline $\begin{array}{l}\text { Create and label areas for various triage } \\
\text { categories }\end{array}$ & $80 \%$ & $20 \%$ & $0 \%$ & $0 \%$ & $0 \%$ \\
\hline
\end{tabular}

Respondents generally agreed $100 \%$ (strongly agree plus agree) for all the possible way forwards to improving on the existing local protocol.

Possible way forward - conduct regular training on triage, Adopt a unified triage scale nationwide, Print triage tool(chart/scales) and put in open place in emergency re- ceiving areas, administrative support in terms of number of staff on duty were the major ways forward to improving triage system. (table 3).

Table 3. Steps taken when a "very sick patient" arrives at the emergency receiving areas in hospitals with no formal triage system

\begin{tabular}{ll}
\hline Hospital & Steps taken when sick patients arrive \\
\hline Anaka hospital & $\begin{array}{l}\text { History/vitals, triage, Clinician's review, registrations and } \\
\text { admission/referral in that order }\end{array}$ \\
$\begin{array}{l}\text { Gulu RRH } \\
\text { and Kitgum hospitals }\end{array}$ & $\begin{array}{l}\text { admission/referral in that order } \\
\text { Triage, registrations, history/vitals, Clinician's review, } \\
\text { admission/referral and billing in that order }\end{array}$ \\
\hline $\begin{array}{l}\text { Kalongo and St. Joseph's } \\
\text { hospital }\end{array}$ & \\
\hline
\end{tabular}

Steps taken when sick patients arrive at emergency receiving areas in hospitals with no formal triage system in place

Settings with no formal triage system in use (using "eye-ball" triage)

All participants in hospitals with no formal triage rated importance of having formal triage system as"very important" and all agreed on possible improved patients' care if formal triage system was to be in place. 
Table 4. Opinion on barriers to development of formal triage system in hospitals with "eyeball" triage system

\begin{tabular}{llllll}
\hline $\begin{array}{l}\text { Barriers to development of Triage } \\
\text { (n= 28) }\end{array}$ & $\begin{array}{l}\text { Strongly } \\
\text { Agree }\end{array}$ & Agree & $\begin{array}{l}\text { Not } \\
\text { Sure }\end{array}$ & Disagree & $\begin{array}{l}\text { Strongly } \\
\text { Disagree }\end{array}$ \\
\hline No national guideline exist on triage & $50 \%$ & $18 \%$ & $32 \%$ & $0 \%$ & $0 \%$ \\
$\begin{array}{l}\text { No triage chart displayed in emergency } \\
\text { receiving areas to guide practice }\end{array}$ & $61 \%$ & $36 \%$ & $4 \%$ & $0 \%$ & $0 \%$ \\
Staff are not trained on triage & $36 \%$ & $36 \%$ & $0 \%$ & $21 \%$ & $7 \%$ \\
\hline $\begin{array}{l}\text { Shortage of staff on duty to perform triage } \\
\text { Triage is considered not very important }\end{array}$ & $57 \%$ & $32 \%$ & $0 \%$ & $11 \%$ & $0 \%$ \\
\hline $\begin{array}{l}\text { Triage wastes time } \\
\text { No space to allocate patients into categories } \\
\text { even if triaged }\end{array}$ & $18 \%$ & $4 \%$ & $7 \%$ & $32 \%$ & $43 \%$ \\
\hline Poor administrative policy and support & $21 \%$ & $29 \%$ & $7 \%$ & $39 \%$ & $7 \%$ \\
\hline
\end{tabular}

Lack of displayed triage protocol, shortage of staff on duty, lack of training on triage and lack of national triage guideline were the common barriers as per participants' opinion while some respondents thought triage waste times and not considered important.

Barriers to development of formal triage system

Lack of national triage guidelines, absence of triage guide- lines displayed in emergency receiving areas and shortage of staff on duty were cited as the main barriers. (table 5).

Table 5. Opinion on possible way forward to develop formal triage systems in hospitals with "eyeball" triage

\begin{tabular}{|c|c|c|c|c|c|}
\hline $\begin{array}{l}\text { Way Forward to develop triage } \\
(\mathrm{n}=\mathbf{2 8})\end{array}$ & $\begin{array}{l}\text { Strongly } \\
\text { Agree }\end{array}$ & Agree & $\begin{array}{l}\text { Not } \\
\text { Sure }\end{array}$ & Disagree & $\begin{array}{l}\text { Strongly } \\
\text { Disagree }\end{array}$ \\
\hline Conduct regular training on triage & $86 \%$ & $14 \%$ & $0 \%$ & $0 \%$ & $0 \%$ \\
\hline Formulate or adopt a triage system/scale & $71 \%$ & $29 \%$ & $0 \%$ & $0 \%$ & $0 \%$ \\
\hline $\begin{array}{l}\text { Need to assign staff specifically to do } \\
\text { triage during each shift }\end{array}$ & $64 \%$ & $29 \%$ & $0 \%$ & $4 \%$ & $4 \%$ \\
\hline $\begin{array}{l}\text { Print triage tool(chart/scales) and put in } \\
\text { open place in emergency receiving areas }\end{array}$ & $82 \%$ & $18 \%$ & $0 \%$ & $0 \%$ & $0 \%$ \\
\hline Administrative support & $68 \%$ & $25 \%$ & $7 \%$ & $0 \%$ & $0 \%$ \\
\hline Create space for triage categories & $57 \%$ & $36 \%$ & $4 \%$ & $4 \%$ & $0 \%$ \\
\hline
\end{tabular}

Participants agreed with all suggested way forward to develop triage protocol with exception of some few disagreed on the point of creating space specifically for triage and assigning a staff specifically to do triage.

Possible way forward to develop formal triage system The majority of participants agreed withall suggested way forward in order to develop triage system. (table 5).

\section{Discussion}

One hospital - Gulu Independent Hospital - declined to grant permission for the survey. However, this does not have significant effect on results as 33 out of expected
38 participants were recruited for the study making up to $86.8 \%$ response rate. In addition, this hospital was a private hospital (although profit oriented) which is still fully represented by the three private hospitals recruited allowing the results to be generalised to the other sub-regions. This study shows that the use of formal ED triage system is very rare in Northern Uganda (only 1 out of 6 hospitals $-16.7 \%$ ). This is far less compared to a report 
by Uganda Malaria Consortium which showed 44\% of health facilities surveyed in Hoima central, Uganda had functional triage ${ }^{12}$. A possible explanation for this difference is that the study in Hoima didn't specify between adult or paediatric triage as there has been training and rapid uptake of WHO - ETAT+ in Uganda ${ }^{9}$ under the package of IMCI. This result is as well low compared to findings in Tanzania, similar settings like Uganda where it was found that $40 \%$ of 10 hospitals surveyed had an adult triage system in place ${ }^{17}$. Over all, these are low figures for effective emergency service delivery at emergency receiving areas. Even though we think that resource scarcity is the explanation for poor emergency services, it may not hold truth in all aspects as having a triage protocol routinely used in emergency receiving areas should not require unaffordable magnitude of resources.

This local protocol uses similar approach in assessing severity of illness and taking vital signs to aid in making triage decisions i.e. airway, breathing, circulation and level of consciousness while taking vitals, although triage per se may not need such approach but this is vital in systematic approach of assessing patients as a whole. This is consistent with most triage protocols used in other countries ${ }^{18,19}$. There is no formal staff training on this triage protocol which raises questions whether nurses are effectively using this protocol or not. It has been found that training improves triage performance ${ }^{20}$ but it's not being done in this setting.

The triage is done by nursing cadres (enrolled and registered nurses) which doesn't differ from what is done elsewhere $^{18}$, however due to shortage of doctors and other non-physician clinicians, these cadres are not involved in triage as might be in other middle and high income countries. It's commendable for this hospital with formal local protocol as this is a very important initiative. It may not matter much which protocol is in use as this varies from country to country and it's difficult to validate which protocol is superior to the other but having one that suits local setting or even better, having one uniform national protocol used countrywide is of paramount importance ${ }^{21}$.

Although most participants rated triage as working well, there have been limitations in improving and using this triage (Table 1). Most notably was the lack of training similar in Tanzania where there was no training on triage, shortage of staff on duty, variations of triage system if present from one hospital to another as well as lack of space. Shortage of staff is a well-known crisis in Africa, especially in sub-Saharan African countries. This is due partly to emigration of health workers to high and middle income countries for economic reasons ${ }^{22}$ and partly due to few numbers of training institutions and low level of output from these training institutions ${ }^{23,24}$. A recent survey which included Uganda, estimated that it would take 29 years for nurses and 36 years for physicians to reach WHO recommendation of 2.28 per 1000 population $^{24}$.

A task-shifting phenomenon where by midlevel emergency care providers trained in emergency care deliver health care to acutely ill patients has been tried in rural Uganda. Nurses underwent two years training program to become emergency care practitioners in rural settings. It was found to have had significant impact on mortality indices in the region compared to data before the implementation of the program ${ }^{25}$. As far as the lack of space/ infrastructure is concerned, it is a general problem in the country similar in other regions as well as other countries like Kenya and Tanzania with similar settings. However, the point here could be inappropriate use of available infrastructure by not prioritizing for emergency services or difficulty in re-arranging the existing infrastructure to suit emergency department strategic locations - need for ambulance disembarkation areas, triage areas, visitors'/ caretakers' waiting areas, etc. Management and staff themselves should assess the feasibility of re-arranging the overall organization of service flow within the available infrastructure which might be easier and achievable within short a term, than demanding for additional totally new infrastructure which is resource intensive given these poor settings.

Triage was done in OPD and Wards in $67.7 \%$ of the hospitals since they don't have dedicated emergency departments. There is scarce published data within the same region if not country wide to compare with this. One study done in 10 hospitals along high ways in Uganda to assess surge capacity in mass casualty incidents found none of these hospitals had dedicated emergency receiving areas ${ }^{26,}$ whereas in Tanzania, 70\% of 10 hospitals surveyed didn't have an emergency room ${ }^{17}$. Using wards during outside routine working hours and OPD during working hours for receiving emergency cases can be complex especially in mass casualty incidents. These areas are often congested and disorganized and can pose great challenges in situation of mass casualties where there is influx of patients, 
attendants, spectators and media teams and this situation can be made worse with the absence of a triage protocol.

It was not surprising to see that all participants concurred with all the possible suggested way forward to improve on the existing local protocol (table 2) - conduct regular training, adopt a unified triage scale nationwide, assign a staff on duty to specifically perform triage, have triage guidelines/chart displayed in an open place in emergency receiving areas and administrative support because these are the simple truths and obvious ways through which improvement can be achieved. However, all these remain opinions without actions unless initiatives are taken by clinicians, researchers and other staff, ministry of health, and individual institution administrations to work towards implementing them.

In the settings with no formal triage, "eyeball" (subjective form of triage) triage was done by nurses (enrolled, registered and in some hospitals nursing assistants) similar to hospital with formal triage protocol. This is not surprising given the scarcity of doctors with more roles in clinical assessment, diagnosis, treatment prescription and evaluation of clinical course.

Anaka hospital seems to have a more logical flow of steps followed when a patient arrives in the emergency receiving areas (table 3) as compared to Gulu RRH \& Kitgum hospitals and Kalong \& St. Joseph's. Since triage is based on mostly vital signs, it is logical to take this first as airway, breathing, circulation and level of consciousness is assessed briefly before triage decision is made as seen in organisation of Anaka hospital. In Gulu RRH, Kitgum, Kalongo and St. Joseph's hospitals reported doing triage first before vitals are taken, which shows that probably triage decisions are not being made basingon vitals, no wonder in these hospitals there are no formal triage protocols and individual staff uses his/her own judgement of how sick a patient appears to be - 'eye ball triage'. Overall organisation of patients' flow in emergency departments has been found to decrease number of patients waiting to be seen and reduced waiting time ${ }^{27}$.

All participants $(100 \%)$ from hospitals with no formal triage accepted in their response the importance of having a formal triage protocol - rated "very important" and acknowledged improvements in patients' outcome in the presence of a formal triage protocol. This is important enough to find that they actually recognise the impor- tance and need for such tools and indirectly informs us how they would be willing to take up and apply a protocol should it be in place.

Just as in the setting with formal triage protocol (discussed above), the main barriers to development of a formal triage system were absence of national guideline that could act as a guide to formulate a local protocol, lack of training, shortage of staff on duty to implement the protocol even if formulated/adopted and poor administrative support (table 4). Overall point picked from this response is that staff do yearn for a triage protocol that somehow would make their work easy and are willing to implement it if present. The main problem at hand is probably lack of initiatives and administrative support and policy to drive the initiatives to create a local protocol at least even if national guidelines do not exist.

This study achieved the set objectives which were to determine presence or absence of a formal triage system, determine the cadre of staff performing triage (formal or "eyeball") and todetermine opinion of staff on barriers to development or improvement of a formal triage system and possible ways through which it can be developed or improved. Findings from this study can be generalised to other regions of Uganda given the similarity of study settings, study participants (all cadres - nurses, physician assistants and doctors) hospitals included (both private and public) and distribution of this hospitals in the region.

\section{Study limitations}

Desirability bias and hurried response at time of data collection could have potentially affected the quality of data collected. However, each staff who felt tired or a bit in a hurry were contacted another time when they felt comfortable and free to participate in the study potentially mitigating the mentioned challenge.

\section{Conclusion}

Adult hospital-based formal triage protocol is lacking in hospitals in Northern Uganda. Most hospitals do not have dedicated emergency receiving areas; emergency patients are received from various wards or OPD. Nursing staff are the main health workers involved in triage - either formal or "eye ball". The main barriers to development/ improvement of triage were lack of national guidelines 
on triage scale, lack of staff training on triage, shortage of staff, lack of space and lack of administrative support and initiatives.

\section{Recommendations}

Ministry of health, using its expertise should develop one formal triage guideline that can be uniformly used in hospitals as well as in training institutions countrywide, individual hospital needs to take initiative and formulate or adopt a triage protocol to be used locally and ministry of health, within its capacity scale up recruitment of health workers - through prioritization as well as scale up training of these cadres in liaison with ministry of Education-increasing the capacity of the available institutions to take more trainees.

\section{Acknowledgement}

We would like to acknowledge the contributions of various people; Staff and administrations of Lacor, Kalongo,St. Joseph's, Gulu regional referral, Anaka and Kitgum hospitals for their co-operations during data collection. Special gratitude goes to the Division of Emergency Medicine and Health Science library for multitude of resources available for developing the proposal and writing this manuscript.

We also extend special gratitude to Total Exploration and Production (E\&P) Uganda who sponsored the whole research project as part of the MPhil scholarship.

\section{Conflict of interest}

This research findings were submitted (in the form of dissertation) by the principle investigator-Dr Keneth Opiro to University of Cape Town in partial fulfilment for the award of the degree-Masters of Philosophy in Emergency Medicine.

The research was sponsored by Total E \& P Uganda to the tune of USD 4,500 as part of the MPhil scholarship. Sponsors had no role in the development of the proposal, conducting the study, statistical analysis as well as writing this final manuscript.

\section{References}

1. Richardson JD, Franklin G, Santos A, Harbrecht B, Danzl D, Coleman R, et al. Effective triage can ameliorate the deleterious effects of delayed transfer of trauma patients from the emergency department to the ICU. Journal of the American College of Surgeons. 2009;208(5):671-8.
2. Rosedale K, Smith Z, Davies H, Wood D. The effectiveness of the South African Triage Score (SATS) in a rural emergency department. SAMJ: South African Medical Journal. 2011;101(8):537-40.

3. Rominski S, Bell SA, Oduro G, Ampong P, Oteng R, Donkor P. The implementation of the South African Triage Score (SATS) in an urban teaching hospital, Ghana. African Journal of Emergency Medicine. 2014;4(2):71-5.

4. Kobusingye OC, Hyder AA, Bishai D, Hicks ER, Mock C, Joshipura M. Emergency medical systems in low-and middle-income countries: recommendations for action. Bulletin of the World Health Organization. 2005;83(8):626-31.

5. Hsia RY, Mbembati NA, Macfarlane S, Kruk ME. Access to emergency and surgical care in sub-Saharan Africa: the infrastructure gap. Health policy and planning. 2012;27(3):234-44.

6. Anthony DR. Promoting emergency medical care systems in the developing world: weighing the costs. Global Public Health. 2011;6(8):906-13.

7. Mufunda J, Chatora R, Ndambakuwa Y, Nyarango P, Kosia A, Chifamba J, et al. Emerging non-communicable disease epidemic in Africa: preventive measures from the WHO Regional Office for Africa. Ethn Dis. 2006;16(2):521-6. PubMed

8. Hyder AA, Labinjo M, Muzaffar SS. A new challenge to child and adolescent survival in urban Africa: an increasing burden of road traffic injuries. Traffic injury prevention. 2006;7(4):381-8.

9. Gove S. Integrated management of childhood illness by outpatient health workers: technical basis and overview. The WHO Working Group on Guidelines for Integrated Management of the Sick Child. Bulletin of the World Health Organization. 1997;75(Suppl 1):7.

10. MacLeod JB, Kobusingye O, Frost C, Lett R, Kirya F, Shulman C. A comparison of the Kampala Trauma score (KTS) with the revised Trauma score (RTS), Injury Severity Score (ISS) and the TRISS method in a Ugandan Trauma registry. European Journal of Trauma. 2003;29(6):392-8. 11. MacLeod J, Kobusingye O, Frost C, Lett R. Kampala Trauma Score (KTS): is it a new triagetool? East and Central African Journal of Surgery. 2007;12(1):74-82.

12. Uganda M. Clinical Audit Uganda - Innovative training improves quality of care Available from: http:// www.malariaconsortium.org/userfiles/file/MC\%20Publications $\% 20$ and $\% 20$ Brochures/Success $\% 20$ story_clinical\%20audit_uganda.pdf. 
13. UNFPA. National Population and Housing Census 2014, Provisional results. Available from: http://unstats.un.org/unsd/demographic/sources/census/2010_ PHC/Uganda/UGA-2014-11.pdf.

14. Göransson K, Ehrenberg A, Ehnfors M, editors. A national survey of emergency department triage in Sweden. AMIA Annual Symposium Proceedings; 2003: American Medical Informatics Association.

15. UNCT. Uganda National Council of Science and Technology 2016 [cited 2016 1st March]. Available from: http://www.uncst.go.ug/.

16. Lacor H. Lacor Hospital Institutional Research and Ethics Committee 2016 [cited 2016 1st March]. Available from: http://www.lacorhospital.org/Researchpublications/Research/LacorHospitalInstitutionalResearchCommittee/tabid/552/Default.aspx.

17. Baker T, Lugazia E, Eriksen J, Mwafongo V, Irestedt L, Konrad D. Emergency and critical care services in Tanzania: a survey of ten hospitals. BMC Health Services Research. 2013;13(1):140.

18. Twomey M, Wallis LA, Thompson ML, Myers JE. The South African Triage Scale (adult version) provides valid acuity ratings when used by doctors and enrolled nursing assistants. African Journal of Emergency Medicine. 2012;2(1):3-12.

19. Jelinek GA, Little M. Inter-rater reliability of the $\mathrm{Na}$ tional Triage Scale over 11,500 simulated occasions of triage. Emergency Medicine. 1996;8(4):226-30. PubMed

20. Sanddal TL, Loyacono T, Sanddal ND. Effect of JumpSTART training on immediate and short-term pediatric triage performance. Pediatric Emergency Care. 2004;20(11):749-53.

21. Twomey M, Wallis LA, Myers JE. Limitations in validating emergency department triage scales. Emergency Medicine Journal. 2007;24(7):477-9.

22. Eastwood JB, Conroy RE, Naicker S, West PA, Tutt
RC, Plange-Rhule J. Loss of health professionals from sub-Saharan Africa: the pivotal role of the UK. The Lancet. 2005;365(9474):1893-900.

23. Connell J, Zurn P, Stilwell B, Awases M, Braichet J-M. Sub-Saharan Africa: Beyond the health worker migration crisis? Social Science \& Medicine. 2007;64(9):1876-91.

24. Kinfu Y, Dal Poz MR, Mercer H, Evans DB. The health worker shortage in Africa: are enough physicians and nurses being trained? Bulletin of the World Health Organization. 2009;87(3):225-30.

25. Chamberlain S, Stolz U, Dreifuss B, Nelson SW, Hammerstedt H, Andinda J, et al. Mortality related to acute illness and injury in rural Uganda: task shifting to improve outcomes. PloS One. 2015;10(4): PubMed e0122559.

26. Onyachi NW, Maniple E, Santini S. Preparedness For Mass Casualties Of Road Traffic Crashes In Uganda: Assessing The Surge Capacity Of Highway General Hospitals. 2011.

27. Miro O, Sanchez M, Espinosa G, Coll-Vinent B, Bragulat E, Milla J. Analysis of patient flow in the emergency department and the effect of an extensive reorganisation. Emergency Medicine Journal. 2003;20(2):143-8.

[T1]More information on the problem statement as indicated by the reviewer.

[T2]More data on the questionnaire as requested by the reviewer.

[T3]Deleted the name of the hospital which declined to offer permission for the research as suggested by the reviewer.

[T4]Title was in the form of question, now changed for uniformity as suggested by the reviewer.

[T5]Table one has been removed and a brief sentence under results put down to stipulate the results on that table. This is in respond to reviewers suggestion to reduce the number of tables. 